\title{
99mTc-MDP Bone Scan In the Detection of Bone Metastases in Breast cancer.
}

\author{
Karan Peepre
}

\begin{abstract}
Skeletal metastases are clinically significant because of associated symptoms, complications such as pathological fractures and their profound significance for staging treatment and prognosis. Detection of bone metastatic lesions allows radiation therapy or surgical interventions to prevent pathological fractures from disabling the patients. High sensitivity of bone scanning in determining the presence and extent of metastatic disease makes extremely important tool in decision making. Bone scans demonstrate metastatic lesions much earlier than X-ray, C.T.,MRI. 99mTechnetium methylene di-phosphonate (99mTc MDP) bone scintigraphy was performed for the detection of bone metastases in histopathologically proven patients of breast cancer. All spot views were taken as the primary method of acquisition, the regions of the skeleton covered by each spot view. The first spot view of the axial skeleton, usually the posterior projection of the chest, was acquired for approximately 500,000 to 1 million counts depending on the field of view (FOV) of the gamma camera. 53 patients of breast cancer were investigated with gamma camera. On visual analysis there was positive scan findings (bone metastases) in 19 patients (35.84\%) and negative scan findings (normal bone scan) in 34 patients (66.6\%). 99mTc-MDP bone scintigraphy elucidates, non-invasively, tumour characteristics and may be indicative for prognosis and response to chemotherapy and hormonal treatment. Purpose of study was to diagnose, staging and see the treatment response in breast cancer patients.
\end{abstract}

Keywords: Bone scan, Bone metastases, Breast cancer, 99mTc-MDP, Skeletal scintigraphy

\section{Introduction}

Radionuclide imaging (RNI) is the branch of Nuclear Medicine concerned with providing diagnostic information about patients following the administration of a radioactive product. Images are produced of the distribution of the radioactive substance within different organs and systems. This can be compared with normal distribution to diagnose if a medical condition is present and assess its extent or severity. The skeletal is one of the most common sites of distant metastasis in many cancers. Bone scan or scintigraphy (BS) using ${ }^{99 m} \mathrm{Tc}-$ methylenediphosphonate (MDP) is considered the most sensitive method of detecting skeletal metastases, and has been used routinely in higher-risk cancer patients, especially in breast, prostate, and lung cancers, which are known for their high incidence rate of bone metastasis(1). The radionuclide bone scan is the cornerstone of skeletal nuclear medicine imaging. Bone scintigraphy is a highly sensitive method for demonstrating disease in bone, often permitting earlier diagnosis or demonstrating more lesions than are found by conventional radiological methods. Primary tumours of bone are relatively rare in adults whereas metastases to bone are very frequent (breast, prostate, lung, head and neck cancer, etc.). This being particularly true for certain primary tumors .Skeletal metastases are clinically significant because of associated symptoms, complications such as pathological fractures and their profound significance for staging treatment and prognosis(2). For the detection and evaluation of bone metastases of various kinds of carcinomas, ${ }^{99 \mathrm{~m}} \mathrm{Tc}$-bone scintigraphy has been used widely because of its overall high sensitivity and the easy evaluation of the entire skeleton(3).

Present scenario involves ${ }^{99 \mathrm{~m}}$ Technetium methylene di-phosphonate (99mTc MDP) bone scintigraphy method of choice for the detection of bone metastases.

To help diagnose pathology of the skeletal system, patients are injected with radiopharmaceuticals composed of Technetium- ${ }^{99 \mathrm{~m}}$ and a bone-seeking molecule such as analogs of calcium, hydroxyl groups or phosphates. In fact the most commonly used molecule is organic diphosphonate bound to Tc- ${ }^{99 m}$. These patients are then scanned by a gamma camera 2-4 hours later; typically, both anterior and posterior scans are taken.

The mechanism behind uptake of the radiodiphosphate is chemisorption (which is adsorption of a molecule to a surface based on chemical bond formation) on the surface of hydroxyapatite crystals of the bone matrix. The determinants of increased uptake are increased skeletal metabolic activity (primarily the extent of osteoblastic activity), the most important factor and accounting for increased uptake at growth centres and centres of osteoblastic and other metabolic activity, increased blood flow and loss of sympathetic tone, which causes vascular dilatation and hyperemia. 


\section{Method:}

This is a prospective study. This study was been performed at the division of Nuclear Medicine, Department of Radiation Oncology, Hamidia\&Kamla Nehru Hospital, Gandhi Medical College, Bhopal, India. Sum of 53 females, 25- 72 years were accounted for the study. All patients were referred for evaluation of suspected recurrence or progression, later in the course of the disease.Out of which 50 females was histopathologically diagnosed as cancer breast. They have been referred to nuclear medicine for bone scan and two patients came directly with the history of lump in breast with pain in joints. All patients underwent wholebody bone planar scintigraphy in the anterior and posterior positions 3 hours after injection before and, after surgery, radiotherapy, chemotherapy and during follow-up. A dose of 22 to $25 \mathrm{mCi}$ 0f ${ }^{99 \mathrm{~m}} \mathrm{Tc}-\mathrm{MDP}$ was injected IV, entire skeleton was taken under the Electronics Corporation of India Limited (ECIL) gamma camera imaging machine equipped with a low-energy high-resolution parallel-hole collimator.All spot views were taken as the primary method of acquisition, the regions of the skeleton covered by each spot view. The first spot view of the axial skeleton, usually the posterior projection of the chest, was acquired for approximately 500,000 to 1 million counts depending on the field of view (FOV) of the gamma camera. The larger the FOV, the larger the number of total counts required to give similar count densities over equivalent regions of the skeleton. Moreover, the presence of physiologically high count density organs (typically the kidneys) may hamper visualization of contiguous structures (typically the spine). Each of the remaining spot views was then acquired for the same time as the first view. Spot images were obtained using a 128.128 or a $256 \times 256$ matrix $(>200,000$ counts).

Reporting: Nuclear medicine physician record appropriate information regarding the patient, especially type of examination, date, radiopharmaceutical (administered activity and route), a summary of patient history, all correlated data from previous diagnostic studies and the clinical problem. Findings, abnormal tracer uptake (increased, decreased, pattern of abnormal uptake, bone findings, soft tissue findings) were noted. We also wrote comparative data (correlation with other diagnostic results and comparison with previous studies in the reports. In Interpretation, a clear diagnosis is given.

\section{Results}

Observations, of the bone scan is very sensitive for localization of skeletal metastases or tumours, but the specificity is low.As per our patients data we found a total of 53 patients of breast cancer were investigated with gamma camera. On visual analysis there was positive scan findings (bone metastases) in 19 patients (35.84 $\%$ ) and negative scan findings (normal bone scan) in 34 patients $(66.6 \%)$. In four patients with available followup data two with a positive ${ }^{99 \mathrm{~m}}$ Tc-MDP scan remained stable and two of three patients with a negative $99 \mathrm{mTc}-$ MDP bone scan had progressive disease.

\section{Discussions:}

Over recent decades, radionuclide bone scan has been used extensively in the evaluation of cancer patients. It provides essential information about the sites of bone lesions (primary and metastatic tumours), their prognosis and the effectiveness of therapy by showing the sequential changes in tracer uptake. ${ }^{99 \mathrm{~m}}$ Technetium methylene diphosphonate ( $99 \mathrm{mTc}$ MDP) bone scintigraphy is currently the method of choice for the detection of bone metastases, but 18F-fluoro-deoxy-D-glucose positron emission tomography (18FDG PET) offers superior spatial resolution and improved sensitivity. We have compared 18FDG PET with 99mTc MDP bone scintigraphy in patients with skeletal metastases from breast cancer and have analyzed the data in subgroups based on radiographic characteristics of lesions. (4).The standard diagnostic method since the 1970's is planar or SPECT scintigraphy using 99mTc labeled polyphosphonates.Studies by Schirrmeister et al. demonstrated that planar bone scintigraphy was $80-90 \%$ sensitive in the detection of peripheral skeletal metastases, but as low as $20-40 \%$ sensitive in the detection of vertebral metastases.Evaluation of metastatic bone disease is possible using FDG PET. FDG accumulates in all cells relative to increased glucose metabolism. Soft tissue as well as bony metastatic sites can demonstrate FDG uptake, making precise anatomic localization of lesions difficult. ${ }^{18} \mathrm{~F} \mathrm{NaFis}$ preferentially deposited at sites of high bone turnover and remodeling, and bone metastases are seen indirectly because uptake depends on skeletal reaction to the tumor. Tracer kinetics depend on both regional blood flow and osteoblastic activity with bone uptake two times higher and blood clearance is faster than $99 \mathrm{mTc}$ labeled polyphosphonates4,resulting in superior bone to background ratio(5).

Radionuclide bone scanning using technetium-labeled polyphosphonates was introduced into clinical practice in the 1970s and was shown to detect BM several months earlier than plain radiographs. Therefore, BS has become one of the most frequently performed nuclear medicine procedures in Europe and the United States. However, the number of BS procedures used in oncology has been reduced because the prevalence of BM in patients with early tumor stages is low and early treatment of metastatic bone disease does not necessarily improve the survival rate. Furthermore, several studies that compared the sensitivity of planar BS with that of MRI have shown that planar BS is less sensitive than previously accepted (6). The spine is a common site of 
bony metastasis. To date, studies have not identified the initial site and pattern of vertebral metastasis in a homogeneous group of patients. Twenty-seven magnetic resonance imaging studies performed on 25 patients with metastatic vertebral breast cancer were reviewed retrospectively. The location and extent of metastatic vertebral involvement were determined. The vertebral body is the most frequent initial site of metastatic seeding. Although radiographically an absent pedicle is often the first sign of metastatic disease, involvement of the pedicle is by direct extension from either the vertebral body or the posterior elements and is therefore a late occurrence in the disease process (7). The combination of functional and morphologic findings with PET/CT increases the diagnostic performance of PET alone (8). Similarly, SPECT/CT promises to overcome the insufficient specificity of planar scintigraphy and SPECT alone (9).SPECT of the spine improved the diagnostic accuracy of bone scans when added to a planar scan in patients with known malignancy and clinical suspicion of spinal metastases when the planar scan was borderline abnormal. It helps in differentiating between benign and malignant lesions of the spine (10). 99mTechnetium methylene diphosphonate (99mTc MDP) bone scintigraphy is currently the method of choice for the detection of bone metastases, but 18F-fluoro-deoxy-D-glucose positron emission tomography (18FDG PET) offers superior spatial resolution and improved sensitivity. We have compared 18FDG PET with 99mTc MDP bone scintigraphy in patients with skeletal metastases from breast cancer. 18FDG PET detected more lesions than 99mTc MDP scintigraphy (mean, 14.1 and 7.8 lesions, respectively; $\mathrm{P}<.01)$. However, $18 \mathrm{FDG}$ detected fewer bone metastases compared with $99 \mathrm{mTc}$ MDP scintigraphy in a subgroup of patients with osteoblastic disease $(\mathrm{P}<.05)$. Higher SUVs were observed for osteolytic than osteoblastic disease (mean, 6.77 and 0.95 , respectively; $\mathrm{P}<.01$ ). Survival was lower in patients with osteolytic disease compared with the remainder $(\mathrm{P}=.01)$. A difference in survival was not found for those patients with high SUVs (> 3.6; $\mathrm{P}=.4)(11)$. Bone scintigraphy is still the work-horse of nuclear medicine in the search for bone metastasis in patients with malignant tumors, especially prostate and breast cancer. Bone scintigraphy is widely available, relatively inexpensive, and highly sensitive in the detection of bone metastasis. The high sensitivity correlates with a lower specificity because many benign conditions, such as degenerative joint disease, infections, and benign bone tumors, exhibit increased uptake of radiotracer ${ }^{(12)}$.Radionuclide imaging is highly sensitive modality with applications in hand wrist conditions(13).

\section{Acknowledgements:}

The authors would like to thank the staff members of the Nuclear medicine Division, department of radiation oncology, Gandhi Medical College, and staff members of Hamidia hospital and Kamla Nehru hospital and other physicians of the city for their valuable help during the imaging acquisition.

Figure: (1) Schematic diagram of Gamma camera

Figure: (2) 99mTc-MDP bone scintigraphy showing increased radio-tracer in the sites of bone metastases in right humerus, right ribs and primary of breast cancer patient.

Figure: (3): 99mTc-MDP bone scintigraphy showing increased radio-tracer in the sites of bone metastases in D-12, L1 and L3 vertebras (arrow) breast cancer patient.

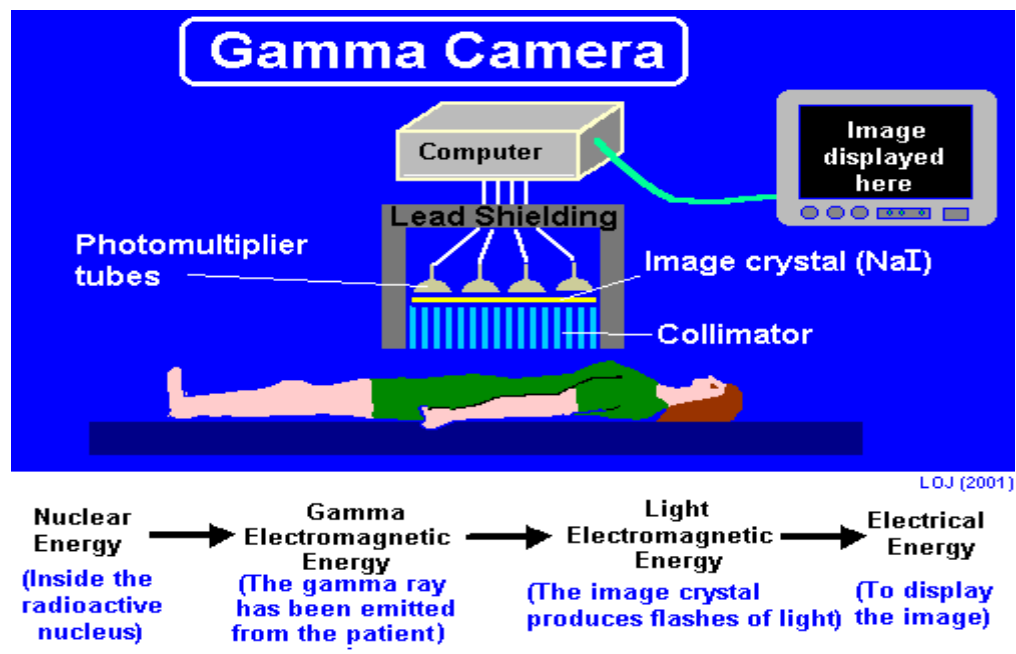

Fig-1: Schematic diagram of Gamma camera showing patient under the detector head and image on computer. 


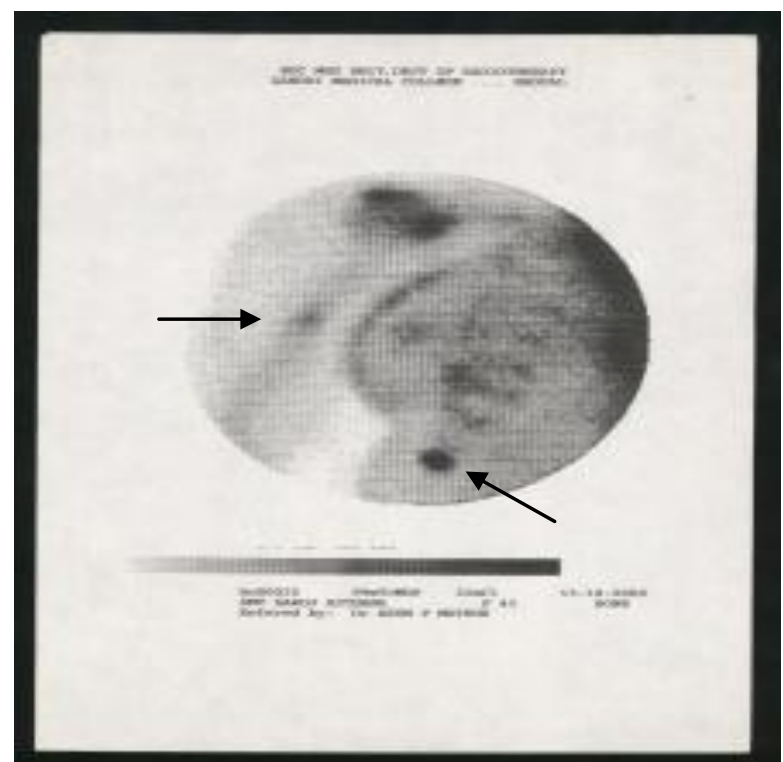

Fig-2: 99mTc-MDP bone scintigraphy showing increased radio-tracer in the sites of bone metastases in right humerus, right ribs (arrow) and primary of breast cancer patient.

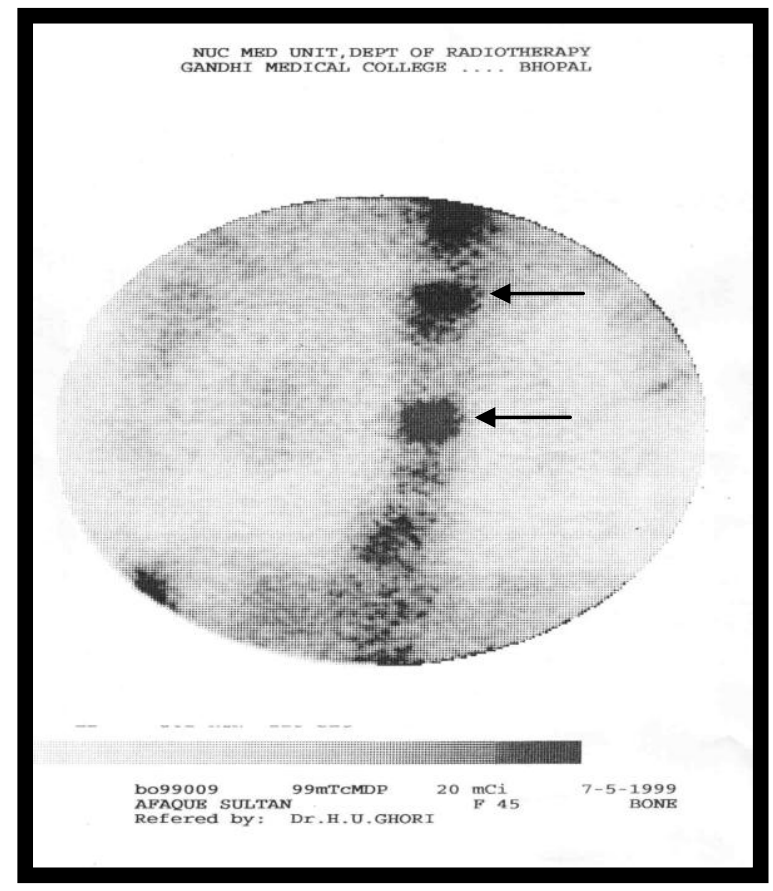

Fig-3: 99mTc-MDP bone scintigraphy showing increased radio-tracer in the sites of bone metastases in D-12, $\mathrm{L} 1$ and L3 vertebras (arrow breast cancer patient.

\section{References:}

[1]. Jacobson AF. Bone scanning in metastatic disease. In: Collier BD Jr, Fogelman I,Rosenthall L, eds.

[2]. Skeletal Nuclear Medicine. St. Louis, MO: Mosby; 1996:87-123.

[3]. L. D. RYBAK, D. I. ROSENTHAL:Radiological imaging for the diagnosis of bone metastases;Q J

[4]. NUCL MED 2001;45:53-64;Vol. 45 - No. 1 -page-53.

[5]. G J Cook, Houston, et al; Detection of bone metastases in breast cancer by 18FDG PET: differing metabolic activity in osteoblastic and osteolytic lesions. 1998 by American Society of Clinical Oncology

[6]. David Halsey, MD and Gustavo Mercier, MD; 18F NaF PET bone imaging vs. planar 99mTc MDP in a patient with breast cancerSeattle Nuclear Medicine, Seattle, WA

[7]. Trillet V, Revel D, Combaret V, et al. Bone marrow metastases in small cell lung cancer: detection with magnetic resonance imaging and monoclonal antibodies. Br J Cancer.1989;60:83-88. Medline

[8]. Asdourian PL, Weidenbaum M, DeWald RL, et al;The pattern of vertebral involvement in metastatic vertebral breast cancer. ClinOrthopRelat Res. 1990 Jan;(250):164-70. [PubMed - indexed for MEDLINE] 
[9]. Lardinois D, Weder W, Hany TF, et al. Staging of non-small-cell lung cancer with integrated positron-emission tomography and computed tomography. N Engl J Med 2003; 348:2500-2507

[10]. SchillaciO, Danieli R, Manni C, Simonetti G. Is SPECT/CT with a hybrid camera useful to improve scintigraphic imaging interpretation? Nucl Med Commun 2004; 25:705-710

[11]. Sedonja I, BudihnaNV;The benefit of SPECT when added to planar scintigraphy in patients with bone metastases in the spine. ClinNucl Med. 1999 Jun;24(6):407-13.

[12]. G J Cook, S Houston,RRubens,et al ;Detection of bone metastases in breast cancer by 18FDG PET: differing metabolic activity in osteoblastic and osteolyticlesions.Journal of Clinical Oncology; 1998 by American Society of Clinical Oncology

[13]. Love C, Din AS, Tomas MB, et al.- Radionuclide bone imaging: an illustrative review. RadioGraphics 2003; 23:341-358 